\title{
Update on once-daily zonisamide monotherapy in partial seizures
}

\author{
This article was published in the following Dove Press journal: \\ Neuropsychiatric Disease and Treatment \\ 19 March 2014 \\ Number of times this article has been viewed
}

\author{
Pegah Afra' \\ Bola Adamolekun ${ }^{2}$ \\ 'Department of Neurology, School \\ of Medicine, University of Utah, \\ Salt Lake City, UT, ${ }^{2}$ Department of \\ Neurology, University of Tennessee \\ Health Science Center, Memphis, \\ TN, USA
}

\begin{abstract}
Zonisamide is an antiepileptic drug that is structurally different from other antiepileptic agents. Its long half-life, once-daily dosing, lack of induction of hepatic enzymes, and broad spectrum of action makes it a suitable candidate for monotherapy. It has been approved as monotherapy for partial onset epilepsy in Japan and South Korea for more than a decade, and was recently approved as monotherapy in Europe. In the USA, it is only approved by the US Food and Drug Administration for adjunctive treatment of partial onset epilepsy. In this paper, we briefly review the literature on zonisamide monotherapy in partial onset epilepsy with regard to its efficacy, safety, tolerability, and long-term side effects, including a recent noninferiority trial in comparison with extended-release carbamazepine. While European regulatory agencies use noninferiority trials for approval of monotherapy, such a trial design does not meet the current regulatory requirements for approval as monotherapy in the USA.
\end{abstract}

Keyword: zonisamide, Zonegran, monotherapy

\section{Introduction}

Zonisamide is a benzisoxazole derivative that is structurally different from other antiepileptic drugs. ${ }^{1}$ It is currently approved in the USA for adjunctive treatment of partial onset epilepsy, but in practice is used as adjunctive treatment for a wide variety of intractable seizures, including the primary generalized epilepsies. ${ }^{2,3}$ It was first marketed in Japan in 1989 for adjunctive treatment of partial epilepsy by Dainippon Pharmaceutical Co, Ltd, under the brand name Excegran ${ }^{\circledR} .{ }^{4}$ Thereafter, Dong-A Pharmaceutical Co, Ltd, launched the product in South Korea in 1992 (also under the brand name Excegran), and Elan Corporation PLC launched it in the USA in 2000 as Zonegran $^{\circledR}{ }^{4}$ In 2004, Eisai obtained manufacturing, development, and marketing rights for Zonegran in the USA and Europe, and subsequently launched the product in Europe in 2005. ${ }^{4}$ Currently, Eisai also markets the product in Asia (including the People's Republic of China, Taiwan, and other countries). ${ }^{4}$

Zonisamide is approved as monotherapy and adjunctive therapy for partial onset and generalized epilepsies in Japan and South Korea, and as monotherapy or adjunctive therapy for partial onset epilepsy in Europe. ${ }^{3,5}$ In the USA, the only indication approved by the US Food and Drug Administration (FDA) for zonisamide is adjunctive treatment of partial onset epilepsy. For monotherapy approval, the FDA requires either a superiority trial using a pseudo-placebo design (since placebo-controlled studies are considered unethical in epilepsy) or a conversion to monotherapy design using historical controls. ${ }^{6,7}$ No such trials exist for zonisamide, so zonisamide currently has no FDA-approved indications for monotherapy in the USA. In this paper, we discuss the efficacy, tolerability,
Correspondence: Pegah Afra Department of Neurology, University of Utah, $175 \mathrm{~N}$ Medical Drive East, 5th Floor, Salt Lake City, UT 84I32, USA Email pegah.afra@hsc.utah.edu 
and safety of zonisamide monotherapy for partial seizures, and examine the obstacles to FDA approval of zonisamide for monotherapy in the USA.

\section{Pharmacokinetic and pharmacodynamic properties}

Zonisamide is a sulfonamide and is chemically similar to an indole. It was originally synthesized in 1972 as one of a series of 1,2-benzisoxazole derivatives by Uno et al in the research laboratories at Dainippon Pharmaceutical Co, Ltd. ${ }^{3,8}$ Due to its anticonvulsant activity in maximal electroshock experiments, it was selected as a candidate for further development as an antiepileptic drug. ${ }^{3}$

Zonisamide is completely absorbed, with a bioavailability of $100 \%$ and reaching maximum plasma concentration in 2-5 hours. ${ }^{1,9}$ Food reduces the rate but not extent of absorption, increasing the time to maximum plasma concentration to 4-6 hours. ${ }^{1,9}$ Zonisamide displays dose-dependent pharmacokinetics, with maximum plasma concentrations and the area under the time-plasma concentration curve reaching linearity after single doses of 100-800 $\mathrm{mg}$ and after multiple doses of 100-400 mg daily. ${ }^{1,10}$ It has a rather long half-life of 60 hours, allowing for once-daily dosing. ${ }^{1,9}$ The maintenance dosage is $8 \mathrm{mg} / \mathrm{kg}$ per day in children and $100-600 \mathrm{mg}$ per day in adults. It is about $40 \%-50 \%$ protein bound, ${ }^{1,9}$ and the therapeutic range is $10-40 \mu \mathrm{g} / \mathrm{mL}$. There is no clear relationship between serum zonisamide levels and clinical efficacy.

Fifty percent of the drug is metabolized in the liver via cytochrome P450 3A4. Enzyme-inducing drugs like carbamazepine, phenytoin, and phenobarbital increase the clearance of zonisamide, leading to decreased plasma levels, although reduction of the elimination half-life of zonisamide does not require a change in the dosing interval for this drug. ${ }^{1}$ Twenty percent of the drug is metabolized by acetylation and $30 \%$ is excreted unchanged in the urine. ${ }^{9,11}$

Zonisamide is a broad-spectrum antiepileptic agent with multiple mechanisms of action. These include reduction of sustained high-frequency repetitive firing of sodiumdependent action potentials, inhibition of low threshold T-type calcium currents, a modulatory effect on GABAmediated neuronal inhibition, inhibition of glutamate release, and weak inhibition of carbonic anhydrase. ${ }^{9}$ The latter may contribute to some of the side effects of this drug. Zonisamide also alters the metabolism of dopamine, 5-hydroxytryptamine, and acetylcholine. ${ }^{9}$

\section{Efficacy of zonisamide as monotherapy}

There have been multiple retrospective chart review studies suggesting the potential efficacy and safety of zonisamide as monotherapy. ${ }^{12-15}$ Park et al found zonisamide monotherapy to be effective across a broad spectrum of seizure disorders, except for complex partial seizures. ${ }^{16}$ In addition, there have been multiple open-label prospective studies of the effectiveness of zonisamide monotherapy in the past two and half decades. Some of these studies are discussed below and are summarized in Table 1.

In a study by Fujii et al in 1989, 25 treatment-naïve patients with cryptogenic, localization-related epilepsy were treated with zonisamide $8 \mathrm{mg} / \mathrm{kg}$ /day once daily for 12 months. In this study, $68 \%$ of patients showed complete seizure control. ${ }^{3,17}$ In a monotherapy trial including 44 pediatric patients (25 with localization-related epilepsy and 13 with generalized epilepsy) reported by Kumagai et al, zonisamide was used in doses up to $12 \mathrm{mg} / \mathrm{kg} /$ day. Seizure freedom was achieved in $72 \%$ of subjects with localizationrelated epilepsy (18/25) and in $91 \%$ of those with generalized epilepsy (12/13). ${ }^{18}$ Hayakawa et al conducted a prospective monotherapy study of zonisamide $2-10 \mathrm{mg} / \mathrm{kg}$ /day in 32 pediatric patients with newly diagnosed partial onset epilepsy and 15 patients with generalized epilepsy. Seizure freedom was achieved in $66 \%$ of patients and another $17 \%$ had a $>50 \%$ reduction in seizure frequency. ${ }^{3,19}$

Yagi and Seki conducted a monotherapy study in 65 refractory pediatric patients with both partial and

Table I Open-label prospective studies of zonisamide monotherapy

\begin{tabular}{|c|c|c|c|c|}
\hline Reference & Type of epilepsy & $\begin{array}{l}\text { Patients, } \mathbf{n} \\
\text { (characteristics) }\end{array}$ & $\begin{array}{l}\text { Zonisamide } \\
\text { dosage }\end{array}$ & $\begin{array}{l}\text { \% LR seizure- } \\
\text { free }\end{array}$ \\
\hline Fujii et $\mathrm{al}^{17}$ & LR & 25 (treatment-naïve) & 8 mg/kg/day & $68 \%$ \\
\hline Kumagai et al ${ }^{18}$ & LR and generalized & 44 (pediatric) & 12 mg/kg/day & $72 \%$ \\
\hline Hayakawa et al ${ }^{19}$ & LR and generalized & 32 (pediatric, newly diagnosed) & $2-10 \mathrm{mg} / \mathrm{kg} / \mathrm{day}$ & $66 \%$ \\
\hline Yagi and Seki ${ }^{20}$ & LR and generalized & 65 (pediatric, refractory) & $6 \mathrm{mg} / \mathrm{kg} /$ day & $*$ \\
\hline Miura ${ }^{21}$ & LR & 72 (pediatric, treatment-naïve) & $8 \mathrm{mg} / \mathrm{kg} /$ day & $79.2 \%$ \\
\hline Seki et $\mathrm{al}^{22}$ & LR and generalized & 68 (pediatric) & up to $12 \mathrm{mg} / \mathrm{kg} /$ day & $82 \%$ \\
\hline
\end{tabular}

Note: $*$ Only $>50 \%$ reduction in seizure frequency was reported (see text under efficacy of zonisamide as monotherapy).

Abbreviation: LR, localization related. 
generalized epilepsy, and $91 \%$ of patients (42 with localization-related epilepsy and eleven with generalized epilepsy) had a $>50 \%$ reduction in seizure frequency. ${ }^{3,20}$ In a monotherapy study reported by Miura, 72 treatment-naïve pediatric patients with localization-related epilepsy received zonisamide at a maintenance dosage of $8 \mathrm{mg} / \mathrm{kg} /$ day for 6-43 months, and seizure control was achieved in $79.2 \%$ of cases. ${ }^{21}$ Seki et al studied 77 pediatric patients with epilepsy in a prospective monotherapy trial. Sixty-eight patients, 44 of whom had localization-related epilepsy, completed the trial and were included in the efficacy analysis; 36 (82\%) of these 44 patients achieved seizure freedom and a further $9 \%$ had a $>50 \%$ reduction in seizure frequency. Of the eleven patients with generalized epilepsy, ten $(91 \%)$ became seizure-free. ${ }^{22}$

In a randomized multicenter trial of pediatric patients with newly diagnosed epilepsy, low-dose (3-4 mg/kg/day) versus high-dose (6-8 mg/kg/day) zonisamide was evaluated. ${ }^{23}$ Of the $90 / 125$ patients who completed the trial, 49/65 were enrolled in the low-dose group and 41/60 were enrolled in the high-dose group. The seizure freedom rate at 6 months was reported to be $63.1 \%(41 / 65)$ in the low-dose group and $57.6 \%(34 / 60)$ in the high-dose group. ${ }^{23}$

In a multicenter, randomized, double-blind, noninferiority Phase III trial of the efficacy of zonisamide monotherapy, adult patients were recruited from 120 epilepsy centers in Europe, Asia, and Australia. This study compared the efficacy of oral zonisamide with that of controlled-release carbamazepine in patients with newly diagnosed partial onset seizures. Over three-quarters of patients, ie, $79.4 \%$ of those on zonisamide versus $83.7 \%$ of those on controlled-release carbamazepine were seizure-free at the end of 26 weeks or more, and the difference between the two groups was considered to be nonsignificant. Seizure freedom was achieved at the lowest target dose in both treatment groups. This study established that zonisamide is not inferior to carbamazepine, and is well tolerated as monotherapy in patients with newly diagnosed partial onset epilepsy. It is interesting that the proportion of patients with complex partial seizures who became seizurefree was higher in carbamazepine group (80/86) than in the zonisamide group (70/91). ${ }^{24}$

\section{Safety and tolerability as monotherapy}

In all prospective open-label trials of zonisamide monotherapy in the pediatric population, zonisamide was well tolerated with mild to moderate side effects. ${ }^{17-23}$ In the randomized, double-blind, noninferiority Phase III trial in adults, the adverse events were similar for carbamazepine and zonisamide. ${ }^{24}$ The safety data are discussed in the two groups as follows.

Treatment-emergent adverse events were reported in $60 \%$ of patients on zonisamide and in $62 \%$ of those on carbamazepine. Treatment-emergent adverse events reported by $\geq 5 \%$ of patients included headache, decreased appetite, weight loss, somnolence, and dizziness, in order of frequency. Treatment-emergent adverse events leading to discontinuation were fatigue, rash, dizziness, and memory impairment, and their incidences were $11 \%$ in the zonisamide group versus $12 \%$ in carbamazepine group (with rash being the most common in the carbamazepine group and fatigue the most common in the zonisamide group; fatigue did not cause any discontinuation in the carbamazepine group). Serious treatment-emergent adverse events were $5 \%$ for zonisamide versus $6 \%$ for carbamazepine.

Treatment-related adverse events were also similar and reported for $36 \%$ of patients in the zonisamide group versus $38 \%$ of those in the carbamazepine group. These were similar in terms of level of severity between the two groups, and were mild ( $22 \%$ for zonisamide and $20 \%$ for carbamazepine), moderate (11\% for zonisamide and $14 \%$ for carbamazepine) to severe (4\% for zonisamide and $4 \%$ for carbamazepine). Serious treatment-related adverse events occurred in $1 \%$ of the zonisamide group and in $2 \%$ of the carbamazepine group.

A pooled analysis of the safety and tolerability of zonisamide as monotherapy or as add-on therapy in elderly patients ( $\geq 65$ years) did not reveal any new or unexpected safety findings in this age group. ${ }^{25}$ The risk of teratogenicity with zonisamide has not been clearly determined, and it is classified by the FDA as a category $\mathrm{C}$ drug.

\section{Long-term tolerability, safety, and efficacy}

In retrospective studies, zonisamide was a safe, effective, and well tolerated treatment option. ${ }^{12,26}$ Zonisamide did not seem to show any decrease in efficacy during long-term use for 6-180 months. ${ }^{27}$ One prospective, randomized, open-label study evaluated the long-term effects of zonisamide on cognition and mood during monotherapy. ${ }^{28}$ In this study, zonisamide did not elicit significant mood changes, but had negative dose-related effects on several cognitive tests, including delayed word recall, Trail Making Test Part B, and verbal fluency. The cognitive side effects were sustained after one year of treatment.

Adult patients completing the abovementioned randomized, double-blind, noninferiority Phase III trial comparing 
zonisamide versus carbamazepine monotherapy entered a long-term extension study. ${ }^{29}$ The results were presented in abstract form at the International Epilepsy Congress in Montreal in 2013. This study included 137 patients in the zonisamide group and 158 patients in the carbamazepine group, the zonisamide dosage was 200-500 mg/day and for carbamazepine was 400-1,200 mg/day. Long-term safety assessments included treatment-emergent adverse events and clinical laboratory parameters. The overall incidence of treatment-emergent adverse events was similar for zonisamide $(52.6 \%)$ versus carbamazepine $(46.2 \%)$; most treatment-emergent adverse events (>95\%) were of mild or moderate intensity, the most commonly reported being decreased weight $(5.8 \%$ versus $0 \%$, respectively) and headache $(4.4 \%$ versus $6.3 \%)$. Incidences of serious treatment-related adverse events and treatment-emergent adverse events leading to withdrawal were low and similar between the groups $(0.7 \%$ versus $1.9 \%$, and $1.5 \%$ versus $0.6 \%$, respectively). There were small to moderate decreases in bicarbonate levels from baseline in the zonisamide group (mean -3.4 mmol/L). Vital signs and physical/neurological examinations identified no safety concerns.

Efficacy assessments included retention and seizure freedom rates. Retention rates for zonisamide versus carbamazepine were generally similar at all time points $(58.4 \%$ versus $61.4 \%, 27.7 \%$ versus $27.8 \%$, and $5.8 \%$ versus $2.5 \%$ at 12 , 18 , and 24 months, respectively; intent-to-treat population). Seizure freedom rates after 24 months of treatment were $32.3 \%$ versus $35.2 \%$ (zonisamide versus carbamazepine; intent-to-treat population).

\section{Discussion}

About $47 \%-50 \%$ of patients with newly diagnosed seizure disorder become seizure-free with the initiation of the first antiepileptic drug and another 13\%-20\% with a second drug or with dual therapy. ${ }^{30-32}$ Although monotherapy and polytherapy have similar efficacy, monotherapy is associated with improved tolerability. Additionally, drugs that need to be taken less frequently have better adherence rates, and for antiepileptic drugs, the likelihood of missing a dose is increased by $27 \%$ each additional time a drug is expected to be taken daily. ${ }^{33}$

Zonisamide, with its long half-life, once-daily dosing, and efficacy at low to medium dosage is a good candidate for monotherapy. ${ }^{1,9}$ Additionally, it has a broad spectrum of action, making it a useful option when the precise diagnosis is in doubt (ie, it is unlikely to cause seizure exacerbation in the primary care setting). ${ }^{1,9}$ Although it is a sodium channel blocker, it does not induce hepatic enzymes and therefore is unlikely to interact with hormonal contraceptives and a variety of other drugs with hepatic metabolism. ${ }^{1,9}$ It has a minimal effect on mood, but is well known to have sustained cognitive side effects. ${ }^{28}$ Additional side effects include weight loss, which may or may not be desirable, and precautions include metabolic acidosis, kidney stones, oligohydrosis, and hyperthermia in pediatric patients, and pregnancy category $\mathrm{C}$ in women. ${ }^{2}$

The zonisamide versus controlled-release carbamazepine noninferiority trial and its long-term extension suggest favorable safety, efficacy, tolerability, and retention profiles for zonisamide, which makes it a desirable monotherapy agent in partial onset epilepsies. ${ }^{24,29}$ Similarly, four pivotal placebocontrolled trials of zonisamide have established its safety, clinical efficacy, and tolerability in adjunctive treatment of refractory partial onset epilepsy. ${ }^{9}$ In 2012, the European Medicines Agency approved zonisamide as monotherapy. Although the European regulatory agencies use noninferiority trials for monotherapy approval, this trial design does not meet the FDA requirement for monotherapy approval in the USA. Currently, the FDA requires either a superiority trial design or a historical control monotherapy design. ${ }^{6,7}$ Of the drugs that are currently approved for monotherapy in the USA, the first-generation antiepileptic drugs were "grandfathered" by the FDA and did not go through the current approval criteria. ${ }^{34}$ These drugs usually cause liver enzyme induction or inhibition resulting in drug-drug interactions. Further, they have long-term side effects, including effects on bone mineral density or bone turnover. Four of the secondgeneration drugs have been approved by the FDA for monotherapy using a superiority trial design with a pseudo-placebo arm. These include oxcarbazepine, felbamate, lamotrigine, and topiramate, with oxcarbazepine and topiramate having FDA approval as initial monotherapy. ${ }^{6}$ After acceptance of historical control conversion to monotherapy design by the FDA, lamotrigine extended-release was the first drug to obtain FDA approval using this trial design. ${ }^{7}$ Lacosamide is currently under review by FDA as a potential second drug using the historical control conversion to monotherapy design. ${ }^{35}$ The International League Against Epilepsy (ILAE) has recommended active-controlled monotherapy trials with a duration of at least 48 weeks and efficacy assessment of seizure freedom at 24 weeks or more. As such, the zonisamide versus controlled-release carbamazepine noninferiority trial and two other trials ${ }^{36,37}$ (levetiracetam versus controlledrelease carbamazepine published in 2007, and pregabalin versus lamotrigine published in 2011) qualify according to 
the ILAE criteria. A recent ILAE review provides evidence that zonisamide has level A efficacy as initial monotherapy for adults with partial onset seizures. ${ }^{38}$ Pending monotherapy trials in accordance with FDA requirements in the USA, the American Epilepsy Society and American Academy of Neurology may take a lead in assessing the evidence on the efficacy, safety, and tolerability of zonisamide monotherapy in partial onset epilepsy.

\section{Disclosure}

PA serves on the speaker's bureau for, has received honoraria from, and been a consultant to UCB Pharma. She receives research support from Sunovion, UCB Pharma, and Cyberonics. BA serves on the speaker's bureaus of Supernus Pharmaceuticals and UCB Pharma. The authors have no other conflicts of interest to declare.

\section{References}

1. Sills G, Brodie M. Pharmacokinetics and drug interactions with zonisamide. Epilepsia. 2007;48:435-441.

2. Zonegran ${ }^{\circledR}$ Prescribing Information. 2012. Available from: http://us.eisai. $\mathrm{com} / \mathrm{wps} / \mathrm{wcm} / \mathrm{connect} /$ Eisai/Home/Our+Products/ZONEGRAN/ Accessed November 30, 2013.

3. Seino M, Leppik IE. Zonisamide. In: Engel J, Pedley TA, editors. Epilepsy: A Comprehensive Text Book. 2nd ed. Philadelphia, PA, USA: Lippincott Williams and Wilkins; 2008.

4. Dainippon Pharmaceutical Co, Ltd. Dainippon Pharmaceutical and Eisai conclude agreement for the development, manufacture and marketing of the anti-epileptic agent zonisamide in Asia. 2005. Available from: http://www.ds-pharma.com/news/prior/dainippon_2005/20050329. html. Accessed November 30, 2013.

5. Dupont S, Stefan H. Zonisamide in clinical practice. Acta Neurol Scand Suppl. 2012;194:29-35.

6. French JA, Wang S, Warnock B, Temkin N. Historical control monotherapy design in the treatment of epilepsy. Epilepsia. 2010;51: 1936-1943.

7. French JA, Temkin NR, Shneker BF, Hammer AE, Caldwell PT, Messenheimer JA. Lamotrigine XR conversion to monotherapy: first study using a historical control group. Neurotherapeutics. 2012;9: 176-184.

8. Uno H, Kurokawa M, Masuda Y, Nishimura H. Studies on 3-substituted 1,2-benzisoxazole derivatives. 6. Syntheses of 3-(sulfamoylmethyl)-1,2benzisoxazole derivatives and their anticonvulsant activities. $J$ Med Chem. 1979;22:180-183.

9. Brodie MJ, Ben-Menachem E, Chouette I, Giorgi L. Zonisamide: its pharmacology, efficacy and safety in clinical trials. Acta Neurol Scand Suppl. 2012;194:19-28.

10. Kochak GM, Page JG, Buchanan RA, Peters R, Padgett CS Steady-state pharmacokinetics of zonisamide, an antiepileptic agent for treatment of refractory complex partial seizures. J Clin Pharmacol. 1998;38:166-171.

11. Shah J, Shellenberger K, Canafax DM. Zonisamide: chemistry, biotransformation, and pharmacokinetics. In: Levy RH, Mattson RH, Meldrum BS, Perucca E, editors. Antiepileptic Drugs. 5th ed. Philadelphia, PA, USA: Lippincott Williams \& Wilkins; 2002.

12. Newmark ME, Dubinsky S. Zonisamide monotherapy in multi-group clinic. Seizure. 2004;13:223-225.

13. Wilfong AA. Zonisamide monotherapy for epilepsy in children and young adults. Pediatr Neurol. 2005;32:77-80.

14. Kim HL, Aldridge J, Rho JM. Clinical experience with zonisamide monotherapy and adjunctive therapy in children with epilepsy at a tertiary care referral center. J Child Neurol. 2005;20:212-219.
15. Kothare SV, Kaleyias J, Mostofi N, et al. Efficacy and safety of zonisamide monotherapy in a cohort of children with epilepsy. Pediatric Neurol. 2006;34:351-354.

16. Park SP, Kim SY, Hwang YH, Suh CK, Kwon SH. Long term efficacy and safety of zonisamide monotherapy in epilepsy patients. $J$ Clin Neurol. 2007;3:175-180.

17. Fujii T, Furukama H, Matsumoto K, et al. [Dainippon Pharmaceutical Co, Ltd, Developmental Laboratories. Data on file, 1989]. Japanese.

18. Kumagai N, Seki T, Yamawaki H, et al. Monotherapy for childhood epilepsies with zonisamide. Jpn J Psychiatry Neurol. 1991;45: 357-359.

19. Hayakawa T, Nejihashi Y, Kishi T, et al. [Serum zonisamide concentration in fresh cases of childhood epilepsy following zonisamide monotherapy]. J Jpn Epilepsy Soc. 1994;12:249-254. Japanese.

20. Yagi K, Seki T. [Zonisamide in Epilepsy Therapy: Remarkable Improvement in 100 Cases]. 1st ed. Tokyo, Japan: Sozo Shuppan; 1994. Japanese.

21. Miura H. Zonisamide monotherapy with once-daily dosing in children with cryptogenic localization-related epilepsies: clinical effects and pharmacokinetic studies. Seizure. 2004;13 Suppl 1:S17-S23.

22. Seki T, Kumagai N, Maezawa M. Effects of zonisamide monotherapy in children with epilepsy. Seizure. 2004;13 Suppl 1:S26-S32.

23. Eun SH, Kim HD, Eun BL, et al. Comparative trial of low-and highdose zonisamide as monotherapy for childhood epilepsy. Seizure. 2011;20:558-563.

24. Baulac M, Brodie MJ, Patten A, Segieth J, Giorgi L. Efficacy and tolerability of zonisamide versus controlled-release carbamazepine for newly diagnosed partial epilepsy: a phase 3 , randomized, double-blind, non-inferiority trial. Lancet Neurol. 2012;11:579-588.

25. Trinka E, Giorgi L, Patten A, Segieth J. Safety and tolerability of zonisamide in elderly patients with epilepsy. Acta Neurol Scand. 2013;128:422-428.

26. Tosches WA, Tisdell J. Long-term efficacy and safety of monotherapy and adjunctive therapy with zonisamide. Epilepsy Behav. 2006;8:522-526.

27. Fukushima K, Seino M. A long-term follow-up of zonisamide monotherapy. Epilepsia. 2006;47:1860-1864.

28. Park SP, Hwang YH, Lee HW, Suh CK, Kwon SH, Lee BI. Long-term cognitive and mood effects of zonisamide monotherapy in epilepsy patients. Epilepsy Behav. 2008;12:102-108.

29. Baulac M, Bagul M, Patten A, Giorgi L. Long-term safety and efficacy of zonisamide versus carbamazepine monotherapy for treatment of partial seizures in adults with newly diagnosed epilepsy: results of a phase III, multinational, randomized, double-blind, active-controlled study. Epilepsia. 2013;54 Suppl 3:S173.

30. Kwan P, Brodie MJ. Epilepsy after the first drug fails: substitution or add on? Seizure. 2000;9:464-468.

31. Brodie MJ, Barry SJ, Bamagous GA, Norrie JD, Kwan P. Patterns of treatment response in newly diagnosed epilepsy. Neurology. 2012;78: $1548-1554$.

32. Kwan P, Sander JW. The natural history of epilepsy: an epidemiological view. J Neurol Neurosurg Psychiatry. 2004;75:1376-1381.

33. Cramer JA, Glassman M, Rienza V. The relationship between poor medication compliance and seizures. Epilepsy Behav. 2002;3: 338-342.

34. St Louis EK, Rosenfeld WE, Bramley T. Antiepileptic drug monotherapy: the initial approach in epilepsy management. Curr Neuropharmacol. 2009;7:77-82.

35. UCB Media Center. UCB files VIMPAT ${ }^{\circledR}$ (lacosamide) in the US as monotherapy treatment in adult epilepsy patients with partial-onset seizures. 2013. Available from: http://www.ucb.presscentre.com/News/ UCB-files-VIMPAT-lacosamide-in-the-US-as-monotherapy-treatmentin-adult-epilepsy-patients-with-pa-46e.aspx. Accessed November 30, 2013.

36. Brodie MJ, Perucca E, Ryvlin P, Ben-Menachem E, Meencke HJ. Comparison of levetiracetam and controlled-release carbamazepine in newly diagnosed epilepsy. Neurology. 2007;68:402-408. 
37. Kwan P, Brodie MJ, Kälviäinen R, Yurkewicz L, Weaver J, Knapp LE. Efficacy and safety of pregabalin versus lamotrigine in patients with newly diagnosed partial seizures: a phase 3, double-blind, randomized, parallel group trial. Lancet Neurol. 2011;10:881-890.
38. Glauser T, Ben-Menachem E, Bourgeois B, et al. Updated ILAE evidence review of antiepileptic drug efficacy and effectiveness as initial monotherapy for epileptic seizures and syndromes. Epilepsia. 2013;54: $551-563$.

\section{Publish your work in this journal}

Neuropsychiatric Disease and Treatment is an international, peerreviewed journal of clinical therapeutics and pharmacology focusing on concise rapid reporting of clinical or pre-clinical studies on a range of neuropsychiatric and neurological disorders. This journal is indexed on PubMed Central, the 'PsycINFO' database and CAS.

The manuscript management system is completely online and includes a very quick and fair peer-review system, which is all easy to use. Visit http://www.dovepress.com/testimonials.php to read real quotes from published authors.

Submit your manuscript here: http://www.dovepress.com/neuropsychiatric-disease-and-treatment-journal 